\title{
Family-focused obesity prevention program implementation in urban versus rural communities: a case study
}

\author{
Colleen Flattum ${ }^{1 *}$, Sarah Friend ${ }^{2}$, Melissa Horning ${ }^{2}$, Rebecca Lindberg ${ }^{3}$, Jennifer Beaudette ${ }^{3}$ and \\ Jayne A. Fulkerson ${ }^{2}$
}

\begin{abstract}
Purpose: Despite public health efforts to reduce childhood obesity, there remains an unequal distribution of obesity among rural and urban children, with higher rates in rural areas. However, few studies have compared differences in program delivery. This paper aims to describe differences between an urban and rural program delivery of a familyfocused, community-based intervention program to prevent and reduce obesity among children.

Methods: This paper uses a case study format to provide a descriptive analysis of similar obesity prevention programs, designed by the same research team, implemented in Minnesota in different settings (i.e., an urban and rural setting) with significant community engagement in the adaptation process. The rural NU-HOME program is compared to HOME-Plus, an urban family-based obesity prevention program for school-aged children.

Results: Community engagement in the adaptation process of an urban program to a rural program confirmed some anticipated program content and delivery similarities while identifying key differences that were necessary for adaptation related to engagement with the community, recruitment and data collection, and intervention delivery.

Discussion: When adapting research-tested programs from urban to rural areas, it is important to identify the modifiable behavioral, social, and environmental factors associated with obesity to ensure the content of effective childhood obesity prevention programs is relevant. Customizing a program to meet the needs of the community may increase reach, engagement, and sustainability. In addition, long-term dissemination of a tailored program may significantly reduce childhood obesity in rural communities and be implemented in other rural settings nationally.
\end{abstract}

Keywords: Children, Rural, Intervention, Obesity, Community

\section{Introduction}

Children living in rural areas have about $20-25 \%$ higher odds for overweight/obesity compared to those living in urban areas of the United States [1-5]. In fact, while nationally $32 \%$ of children are overweight, the rate among rural children is $39 \%$ [6-9]. Children who are overweight

\footnotetext{
* Correspondence: flatt018@umn.edu

'Division of Epidemiology and Community Health, School of Public Health, University of Minnesota, 1300 S. 2nd St., Suite 300, Minneapolis, MN 55454, USA

Full list of author information is available at the end of the article
}

are at high-risk for continued obesity and for heart disease, diabetes, cancer and osteoarthritis as adults [3,10-12].

Because of the higher percentages of obesity in rural areas and since childhood obesity prevention programs are scarce in rural settings [12-16], more research is needed to test family-focused, obesity-prevention interventions in rural communities. When translating research tested programs from urban areas to rural areas, it is imperative to identify the specific modifiable behavioral, social, and environmental factors (e.g., geography, food availability, transportation) associated with obesity

(c) The Author(s). 2021 Open Access This article is licensed under a Creative Commons Attribution 4.0 International License, which permits use, sharing, adaptation, distribution and reproduction in any medium or format, as long as you give appropriate credit to the original author(s) and the source, provide a link to the Creative Commons licence, and indicate if changes were made. The images or other third party material in this article are included in the article's Creative Commons licence, unless indicated otherwise in a credit line to the material. If material is not included in the article's Creative Commons licence and your intended use is not permitted by statutory regulation or exceeds the permitted use, you will need to obtain permission directly from the copyright holder. To view a copy of this licence, visit http://creativecommons.org/licenses/by/4.0/ The Creative Commons Public Domain Dedication waiver (http://creativecommons.org/publicdomain/zero/1.0/) applies to the data made available in this article, unless otherwise stated in a credit line to the data. 
in a given community to ensure the content of effective childhood obesity prevention programs is relevant [1721]. Furthermore, while tailored content is a well-known and essential aspect of research intervention programs [22-27], it is critical to consider other research implementation or logistical differences that may exist between urban and rural communities [16].

There tends to be a large gap between knowledge generated through research and the generalizability of that research to other community settings [23, 28-33]. Too often, researchers do not translate or disseminate their research for use in other settings where it is likely to have positive impacts. For example, translating research from urban to rural areas may be an important goal, but best practices for such translation are lacking. Therefore, the aim of this paper is to use a case study format to provide guidance and describe important program content and logistics differences (e.g., recruitment methods, data collection methods, and consideration of a "rural lifestyle" (seasonal activities)) that may require consideration when translating family-focused, childhood obesity prevention interventions developed in an urban setting to a rural setting.

\section{Methods}

The Healthy Home Offerings via the Mealtime Environment (HOME) Plus randomized controlled trial tested the impact of a dynamic intervention program aiming to prevent childhood obesity in a metropolitan area of Minnesota in 2011-2014. The intervention program was developed to actively engage school-age children (8-12 years old) and their parents to promote regular family meals and teach cooking skills and healthful eating as outlined in Table 1. The HOME Plus study design,

Table 1 Session-At-A-Glance: Intervention Program Activities for the Urban HOME Plus and Rural NU-HOME Studies

Welcome

Discussion - Parents and Kids

Taste Test

Fruit or *veggie of the month

Food Preparation - Parents and Kids

Discussion - Separate Groups

Parent Group

Kid Group

Hands on activity - Separate Groups

Parent Group

Kid Group

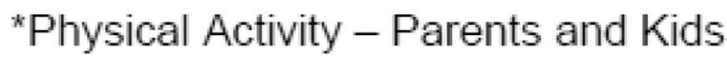

Eat meal (including small children)

Summary and Next Steps
10 minutes

5 minutes

5 minutes

25 minutes

Up to 15 minutes

Up to 25 minutes

Up to 10 minutes

(time taken from Hands-

on Activity Time)

25 minutes

5-10 minutes

120 minutes 
methods, eligibility, data collection information, and intervention description are published in detail elsewhere [34-37]. The urban HOME Plus intervention, delivered in a rural setting, produced a promising reduction in excess weight gain for those that had not started puberty [38]. Meanwhile, the Heart of New Ulm Project (HONU), a population-based demonstration project designed to reduce modifiable cardiovascular (CVD) risk factors in adults was underway in the rural community of New Ulm, MN approximately 95 miles from the Minneapolis metropolitan area [39-41]. Through university-community networking, these two groups decided to extend the adult-focused rural community programming of HONU to include child- and familyfocused obesity prevention programming due to the request of the community. Thus, these two groups collaborated to write and receive funding from the National Institutes of Health to translate the urban-based HOME Plus program to a rural Minnesota community in a randomized controlled trial called the New Ulm at HOME (NU-HOME) study in 2016-2019.

Like the urban HOME Plus study, the primary objective of the rural NU-HOME study was to prevent childhood obesity. Although considerable preliminary work with community partners was conducted for both the urban and rural settings, the collaborative effort between the HONU team and UMN researchers allowed for a deeper understanding of the unique needs of a rural environment [42]. To adapt the intervention, the NUHOME research team worked intensely with a NUHOME Community Action Team (i.e., staff from the local health system, schools, public health departments, and other primary stakeholders who were already engaged with community health improvements through the HONU programs). These community stakeholders were consulted about the greatest needs of the community to ensure the program would have the highest potential for impact. Community Action Team members worked to create and revise a logic model that guided program revisions. The Community Action Team met bimonthly to discuss recruitment, retention, and the intervention content and focus.

The resulting session activities followed the same format for both the urban HOME Plus and rural NUHOME studies (Table 1). The intervention program content was also generally quite similar with the exception of physical activity, which was added for the rural NU-HOME program (Table 2). While the program content was similar in both interventions, in NU-HOME, a few modifications were made, including reduced monthly sessions from 10 to 7 (to fit within the academic school year), and intervention messages were consolidated and reinforced throughout the program (Table 2). Both programs invited all participating family members to attend the sessions, and childcare was provided for younger siblings (2-6 year olds) to remove barriers to participation. The NU-HOME study targeted a younger population of 7-10 year old children and their parents, given the importance of pubertal timing for the HOME Plus outcomes.

To facilitate program adaptation of an urban obesity prevention intervention to a rural community, the research team had multiple meetings with community partners to increase the likelihood of a successful trial in the rural setting. Key changes were documented in meeting minutes, observations made in the field, process data collection (e.g., number of children in childcare), and the intervention delivery guide. The following case study describes program content, logistic, and delivery adaptations that were processed with community partners and describes how the rural program differed from the urban program in the end. We review these differences deemed specific to working in a rural community below in order to support future research in rural communities to prevent childhood obesity.

\section{Findings \\ Research adaptations for the rural NU-HOME program Engagement with the community}

Although the NU-HOME study research team and collaborators anticipated some changes in the delivery of an obesity prevention program in a rural setting based on HOME Plus study findings $[35,37,38]$ and existing literature regarding implementing interventions in rural communities [43, 44], it was necessary to engage with the NU-HOME Community Action Team to verify or reject these anticipated changes as well as explore other modifications. Table 3 depicts logistical modifications that were discussed as we adapted the program from an urban to rural setting.

Local support from key-stakeholders is essential for any community-based program [45-48]. In a tight-knit rural community, however, it is especially important to get buy-in and support from the community up front in order to engage and meet the needs of the community $[30,49,50]$. A large percentage of the work during the first year of the rural NU-HOME grant was focused on fostering and building relationships with key stakeholders in the rural community and getting their input on logistics and relevant intervention content [42].

The NU-HOME study research team built upon the existing HONU community partnerships. Finding and working within existing community working groups and, when necessary, establishing new working groups was instrumental in program success. Existing relationships with community partners [HONU] helped facilitate referrals to the study, aided in recruitment, and assisted 
Table 2 Urban HOME Plus and Rural NU-HOME Intervention Session Content

\begin{tabular}{|c|c|}
\hline Urban HOME Plus & Rural NU-HOME \\
\hline $\mathrm{N}=160$ & $\mathrm{~N}=114$ \\
\hline Ages $8-12$ years old & Ages $7-10$ years old \\
\hline $2011-2015$ & $2016-2020$ \\
\hline \multicolumn{2}{|c|}{ Session Topic (delivered through education and discussion), Hands-On Activities are italicized } \\
\hline $\begin{array}{l}\text { 1- Family meals } \\
\text { Wash, chop, slice and kitchen safety }\end{array}$ & $\begin{array}{l}\text { 1- Family meals and physical activity } \\
\text { Wash, chop, slice and kitchen safety } \\
\text { Physical Activity }\end{array}$ \\
\hline $\begin{array}{l}\text { 2- Meal and snack planning } \\
\text { Recipe revolution }\end{array}$ & $\begin{array}{l}\text { 2- Meal and snack planning } \\
\text { Recipe revolution } \\
\text { Physical Activity }\end{array}$ \\
\hline $\begin{array}{l}\text { 3- Portion vs. serving size } \\
\text { Reading food labels }\end{array}$ & $\begin{array}{l}\text { 3- Portion vs. serving size } \\
\text { Reading food labels } \\
\text { Physical Activity }\end{array}$ \\
\hline $\begin{array}{l}\text { 4- Reducing sedentary behavior } \\
\text { Reducing screen time }\end{array}$ & $\begin{array}{l}\text { 4- Reducing high-fat and high-sugar snacks } \\
\text { Strategies to reduce high-fat and high-sugar snacks } \\
\text { and screen time } \\
\text { Physical Activity }\end{array}$ \\
\hline $\begin{array}{l}\text { 5- Sip smarter - the bottom line on sugary drinks } \\
\text { Strategies to reduce sugar sweetened beverages }\end{array}$ & $\begin{array}{l}\text { 5- Sip smarter - the bottom line on sugary drinks } \\
\text { Strategies to reduce sugar sweetened beverages } \\
\text { Physical Activity }\end{array}$ \\
\hline $\begin{array}{l}\text { 6- Increasing fruit and vegetable intake } \\
\text { Increasing fruit and vegetable availability and intake }\end{array}$ & $\begin{array}{l}\text { 6- Fast food } \\
\text { Selecting healthy fast food options \& Increasing fruit } \\
\text { and vegetable availability and intake } \\
\text { Physical Activity }\end{array}$ \\
\hline $\begin{array}{l}\text { 7- Identify changes made } \\
\text { Strategies to maintain goals }\end{array}$ & $\begin{array}{l}\text { 7- Identify changes made } \\
\text { Strategies to maintain goals } \\
\text { Physical Activity }\end{array}$ \\
\hline \multicolumn{2}{|l|}{$\begin{array}{l}\text { 8- Less sugar and fat-a sweet deal } \\
\text { Strategies to reduce high-fat and high-sugar snacks }\end{array}$} \\
\hline \multicolumn{2}{|l|}{$\begin{array}{l}\text { 9- Picking seasonal produce } \\
\text { Strategies when picking seasonal produce for use in } \\
\text { meals and for snacks }\end{array}$} \\
\hline
\end{tabular}

with providing locations to hold data collection activities and intervention sessions.

\section{Intervention content}

Key session topics from the HOME Plus study intervention were presented and discussed with the Community Action Team using interactive assessments such as rating topic importance and prioritization and review and revision of a logic model. Without exception, all topics were deemed necessary for the adapted NU-HOME program. As shown in Table 2, session content for both programs was similar with a focus on family meals, the home food environment, portion sizes (of energy-dense foods) at meals and snacks, the healthfulness of family meals, self-efficacy for meal planning, cooking skill development and sedentary behaviors/screen time, all logical targets for obesity prevention. Needed changes in program content for rural NU-HOME included an added physical activity component due to reliance on automobiles to meet transportation needs, rather than biking or walking, and the evidence for multipronged interventions in obesity prevention science [51], development of a website for additional accessibility to study materials and supplemental information, and increased synergy with existing community programs and resources with the promotion of local and free events. Lessons learned from the HOME Plus study also indicated a focus on vegetables only for taste testing rather than fruit, removal of dessert (even fruit-based desserts) at sessions, targeted discussions of limited access to healthy and affordable food, and resources on food canning and storage relative to quantity and quality of available food and financial resources. Given the consistency of program content between the urban and rural programs, 
Table 3 Logistical Considerations for Adapting a Family-Focused Obesity Prevention Intervention from an Urban to Rural Setting

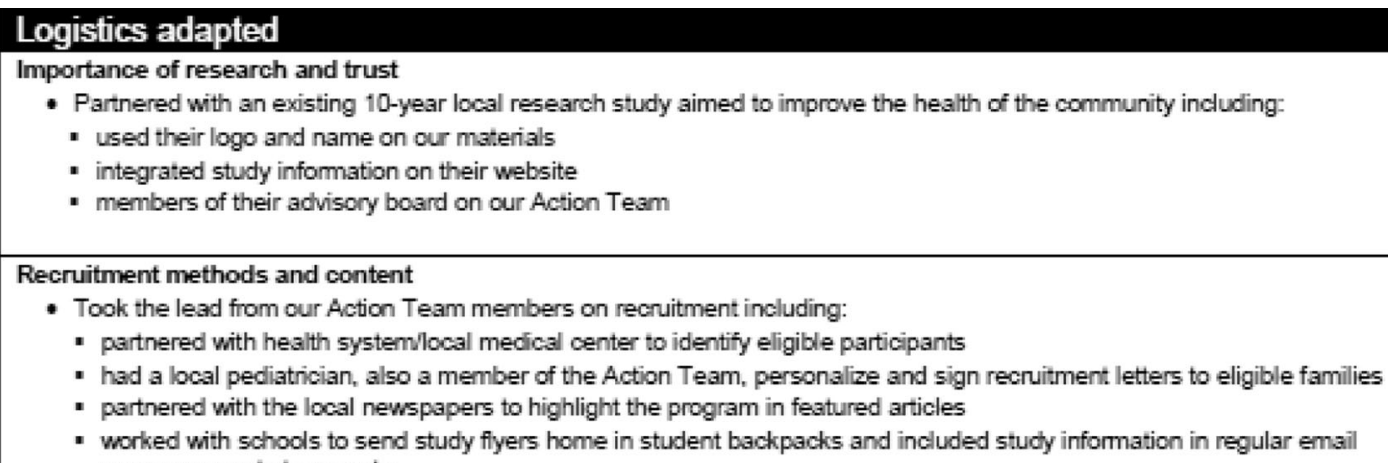

Online session content via website

- Provided access to program materials and recipes via website

Assessment of contamination across treatment groups

- Frequent reminders to not share program materials with those in comparison group who may also be a neighbor, close friend or family member

- Provided opportunity for delayed intervention program to comparison group

Reimbursement for travel to sessions

- Provided gift cards to local gas stations due to the distance from residence to tom for program sessions and activities

the focus of this manuscript is on the logistical program delivery changes that were necessary to successfully implement the trial in an effort to inform future research.

\section{Recruitment and data collection}

Community partnerships within the rural setting were essential to successful recruitment and data collection implementation of the NU-HOME trial. In particular, the primary health care system, schools, public health department and other stakeholders convened regularly and were highly engaged in community health improvements through HONU and we extended this engagement to the NU-HOME study activities. Common recruitment methods for family-based programs (e.g., fliers at community centers, health clinics, schools) were met with support from our community partners. This acceptance was most likely because the previous HONU program work paved the way for a greater understanding of research, expectations, etc. The rural NU-HOME study was able to build on these established and trusted relationships, which enhanced recruitment efforts. For example, direct contact between health care providers and potential study participants (through a letter and at in-person appointments) helped considerably with over half of enrolled parents reporting they heard about the study from their health care provider. The local newspapers in the two participating communities (with a large readership) ran an article about the study free of charge, and the school superintendents at several area schools gave approval to send notices home in students' backpacks and through weekly communications with parents without extensive delays. This community buyin, support and network allowed for an efficient, effective and timely recruitment process. In addition, the school superintendents and principals offered free use of space for data collection, intervention sessions and supply storage, which is less common in more urban programs.

Members of the New Ulm rural community were accustomed to data collection and survey completion since they had experience and familiarity with the HONU project. Yet, challenges were present in the rural community 
that had not occurred in our urban settings. Terms and phrases were different between the urban and rural settings that were relevant for our family meals-focused study. For example, "dinner" in an urban setting is widely accepted to mean "the evening meal." However, in the rural setting with the NU-HOME trial, many community members referred to the midday meal as "dinner" while "supper" was the term for the evening meal. Given that the rural NU-HOME intervention focused on promoting family meals, and in particular, the family evening meal, it was critical to adopt these local terms/phrases in research surveys and intervention delivery for clarity. In addition, in the urban HOME Plus study, only one child per family was eligible for data collection participation and the average number of children per household was two, with typically only one child in the eligible age range. For the rural NU-HOME study, again only one child per family was eligible for data collection participation. However, during the feasibility assessment of potentially eligible children for the rural NU-HOME study (one per participating family), estimates of potentially eligible children from school enrollment figures were overestimated, as children in the rural communities had more siblings and many families had multiple children in the eligible age range. This resulted in two challenges: 1 ) the need for a protocol for selecting one child in a family when more than one was eligible, and 2) a more restricted pool of families to conduct recruitment that affected meeting recruitment goals. The latter challenge required the research team to expand to several nearby rural communities to meet recruitment goals.

\section{Intervention delivery}

Transportation When community-based intervention programs are offered in an urban setting as in the urban HOME Plus trial, it is common to offer transportation assistance to data collection activities and intervention sessions with bus passes or by providing taxi service. However, in this (and many) rural settings, no alternate methods of transportation were available (e.g., no city buses or taxis were in service within the rural communities). Thus, the research team did not have many options for providing transportation assistance. Yet, participating families were used to these transportation norms, and once the sessions began, families connected with one another to arrange carpools, if needed. A different transportation issue was also evident when we expanded recruitment beyond New Ulm, MN to include surrounding communities. Rather than living within walking distance or relatively close to our data collection and intervention sites, many of these families had to drive up to $30 \mathrm{~min} / \mathrm{miles}$ one way to attend data collection activities or intervention sessions. In order to help alleviate the burden of driving such a distance to participate in the intervention, a $\$ 10$ gas gift card was given to each family for each session to help offset transportation costs.

Weather The longer drives also created challenges in the Midwest winter, as traversing a few blocks or miles in poorer weather conditions in urban areas is different from traveling $30 \mathrm{~min} / \mathrm{miles}$ on open road rural areas. In fact, not one urban HOME Plus study intervention session over two years was rescheduled due to weather, while the rural NU-HOME trial had four sessions canceled and rescheduled due to weather. To guide decisions on when cancellations were appropriate, we followed the lead of the local school district and canceled sessions when school activities were canceled due to weather; this protocol was easily communicated and understood by participating families. To address potential barriers around transportation and weather, we created a rural NU-HOME website with study materials and supplemental information for each session that families could access. Although there was not tremendous activity on the website, it offered an alternative method to receive materials if needed [52], [42].

Grocery shopping Each NU-HOME program session included preparing a full meal for all who attended (see Table 1) including entrees and multiple side dishes, requiring a large amount of groceries for each session in both HOME Plus and NU-HOME trials. Grocery delivery is commonly available in urban settings and was used for the urban HOME Plus study. In contrast, grocery delivery was not an option for the rural NU-HOME study as was identified through community-based collaborations early in the process. Alternative arrangements were therefore made with a local grocery store that included sending the grocery list in advance and then interventionists picked up the groceries after they were assembled by store staff. In addition, not all rural communities have access to grocery stores that sell high quality ingredients such as fresh produce at reasonable prices. While the direct New Ulm community had grocery store access with high quality ingredients at reasonable prices, another neighboring community involved in the program did not have access to these resources. Therefore, groceries were often purchased in the New Ulm community for sessions delivered in a nearby community. These resources issues had direct implications for study staff workload and program logistics.

Family size As mentioned previously, the rural NUHOME study families had more children than the families in the urban HOME Plus study, which affected 
intervention delivery. Urban HOME Plus intervention sessions typically had 5-6 participating families, (e.g., one parent, primary child, maybe a sibling) for a total of 15-18 people; however, with larger family sizes in the rural NU-HOME study, sessions frequently had up to 24 attendees per session. As a result, the kitchen space in the rural community needed to be larger, the interventionists need to adapt to engaging more people at one time, and childcare was more heavily utilized at each of the sessions for siblings aged 2-6 years old when compared to the urban setting.

Local events and customs Compared to an urban setting, in the rural setting of the NU-HOME trial, community events often drew a larger proportion of community members, including whole families. For example, school plays, football games, town celebrations (e.g., Octoberfest) and even funerals greatly influenced attendance at NU-HOME intervention sessions, as most of the participants attended the same schools and the communities were very connected. In addition, farming schedules, hunting season, teacher conferences, and religious events were a priority for many families, and it was necessary for the research team to carefully avoid these dates when scheduling study related activities. These events were not as logistically challenging when scheduling the HOME Plus sessions in the urban community.

Treatment group contamination In the rural NUHOME study, the community was smaller than the urban community setting of the HOME Plus study. As such, the smaller community often meant that participants were neighbors, coworkers, close friends or relatives, and therefore, there was an increased risk for contamination (e.g., intervention participants sharing session information with control participants) which could lead to unintended behavior change among control group members. Therefore, intervention staff discussed with families in the intervention group the importance of the randomization process and reiterated the importance at each session not to share information with those outside of the intervention group during the trial.

Delayed intervention programming As part of the urban HOME Plus study design, participating families randomized to the control group received a monthly newsletter. In early discussions with members of the rural community, there was less acceptance among stakeholders of randomization to a "newsletter" control group. Community stakeholders believed all families would be interested in participating in the rural NUHOME hands-on family cooking and physical activity program. Therefore, the study design was adapted in the funded grant proposal to include a "waitlist control" (e.g., the comparison group would participate in intervention sessions upon completion of the study). The delayed intervention required significantly more funding, staffing, and organization than delivering newsletters, but it was deemed essential for study success.

Recipe selection and adjustments to reduce costs In both the urban and rural settings for the HOME Plus and NU-HOME studies, respectively, efforts were made to be mindful of ingredient availability and cost when selecting recipes to be promoted in the intervention program. Thus, session recipes included as many seasonal fruits and vegetables as possible and did not include items with limited availability. These considerations allowed families to purchase recipe ingredients at the local farmer's market(s) or use produce from community or personal gardens.

Differences in cost between urban and rural settings were also seen for mileage and fuel cost for study staff. While some study staff already lived and worked in the rural communities where the NU-HOME study took place, some staff needed to drive from urban areas to deliver the intervention and conduct data collection. These were additional study expenses. Considerable travel costs need to be considered when conducting an intervention in a rural setting if study staff need to travel further distances to conduct study business (i.e., they do not live in or nearby the community given the location of the home institution/university).

\section{Discussion}

Review of recent literature reveals that rural children are at greater risk for obesity than their urban peers $[6,15]$ and previous studies involving rural families have been limited in scope and not specifically designed for the needs, interests, benefits and concerns of rural families $[14,17,18,53]$. Rural families face a number of unique challenges in an attempt to maintain behaviors to promote health and prevent obesity [54]. Such potential challenges include accessibility (e.g., lower availability of fresh fruits, vegetables and other recipe ingredients; travel distances to major grocery stores, exercise facilities, hospitals and clinics, schools and even neighbors are often great distances from their homes), lower incomes as many rural families have limited or seasonal earnings, and limited access to high-speed communications [55]. Therefore, to be an effective obesity prevention program in rural communities, intervention programs must be developed with these potential challenges in mind and must be tailored to the local context and setting. It is important to note that much of the intervention content did not change when adapting our childhood obesity prevention program from an urban setting to a rural setting (likely because the drivers of 
obesity are similar across settings); however, there were many important logistical changes needed in program delivery for the rural setting.

Effective obesity prevention programs for rural families demand unique recruitment and program delivery methods. First, collaboration with key-stakeholders is necessary in order to engage and meet the needs of the community. Stakeholders are particularly well suited to provide insight since they live in the community and are intimately familiar with their residents, including their needs, interests and the concerns as those noted above. Second, the communities participating in the rural NUHOME study had experience with successful recruitment and data collection implementation due to the previous groundwork of the HONU program that paved the way for research in this community. Third, the communitybased NU-HOME program targeted changes in the places where families live, learn, work, and play and provided multiple levels of influence including individual behaviors, family settings, and community institutions.

Given the research considerations related to NUHOME program delivery within a rural setting, guided by the HONU project and the urban HOME Plus study, we delivered a unique family-focused intervention designed specifically for rural families. By developing our NU-HOME program, our hope is that this intervention has the potential to be applied and broadly implemented throughout rural communities to increase knowledge and build skills that could lead to behavior change and eventually health equity for rural youth. The rural NUHOME program also showcases important differences in delivering a family-focused, community-based obesity prevention program from an urban to rural setting.

While this case study has several strengths in how it showcases important differences between translating an urban program to a rural community, a limitation of this work is that an implementation science framework was not applied and could have provided excellent structure for program adaptations. Unfortunately, the timing of NU-HOME study funding and adaptation of the intervention program from the urban HOME Plus study was occurring right before the implementation science field was just coming into its prime. Thus, although our adaptation work was based on existing high-quality translation methodologies, we recommend future intervention adaptations use an implementation science framework for guidance [33, 56-58]. We believe our case study approach informs the field moving forward by providing a base of information for program adaptations for families living in rural communities given the dearth of literature in this area to date.

Public health agencies may find that by forming community joint ventures with schools, healthcare groups, local private sector businesses, and the community, they can focus their mission and create opportunities for healthy living and reduce obesity in rural communities. No single intervention alone will resolve the problem of rural community obesity. However, when a multi-phase approach of activities and programs are offered together, they can encourage and develop lifestyle modifications that encourage healthy behaviors and reduce rural obesity. The collaboration of our research team with existing community organizations that had a similar health promotion mindset, goals, and infrastructure development provides an excellent model for future interventions in rural communities.

This paper highlights specific differences in program delivery between urban and rural community engagement, recruitment and data collection, and intervention programming. This information is particularly useful for others in the early stages of introducing obesity prevention interventions and is broadly informative for programs and funders working in rural areas or those who wish to translate programs developed in urban areas to rural areas.

\begin{abstract}
Acknowledgements
The authors wish to thank the children and families who participated in the NU-HOME study. The authors are also very appreciative of the following interventionists and support staff for their expertise and professionalism in intervention implementation: Kate Callahan Schmitz, Rachel Jones, Shawn Hildebrandt, and nursing students from the Minnesota State University at Mankato. Thank you to the generosity and flexibility of the New UIm and Sleepy Eye School Districts Superintendents for their support of the study and use of the school district facilities. We also thank the following research team members and student volunteers who contributed to the study: Jennifer Linde, Daheia Barr-Anderson, Abbey Sidebottom, Lori Rathburn, Yazmin Cespedes, Jessica Ramos, Christie Martin, Eydie Kramer, Sam Sommerness, Stephanie Grace, and Amanda Folk.
\end{abstract}

\section{Authors' contributions}

JF and RL conceptualized and designed the study. CF, MH, RL, and JA developed the intervention content. SF and JA designed the data collection instruments and SF coordinated and supervised data collection. JB delivered the intervention with oversight by RL. The paper was drafted by CF and was commented on by all authors (SF, MH, RL, JB, JF), who approved the final version. All authors read and approved the final manuscript. Reviewer's comments were reviewed by all authors, revisions drafted by CF and was commented on by all authors (SF, MH, RL, JB, JF), who approved the resubmission of the manuscript.

\section{Funding}

This study was supported by National Institutes of Health $(\mathrm{NIH})$ awards 1R01HL123699 (National Heart, Lung, and Blood Institute; NHLBI) and UL1TR002494 (National Center for Advancing Translational Sciences; NCATS). The content is solely the responsibility of the authors and does not necessarily represent the official views of the NHLBI, the NCATS or the NIH.

Availability of data and materials

The data analyzed during the current study is available from the corresponding author on reasonable request.

\section{Declarations}

Ethics approval and consent to participate

The project was conducted in accordance with relevant quidelines and regulations between the Quorum Review Institutional Review Board (IRB), an external IRB contracted to review on behalf of Allina Health, and the Institutional Review Board at the UMN. The HOME Plus study was reviewed 
and approved by the University of Minnesota's Institutional Research Board. All experimental protocols for the NU HOME study were approved by both the University of Minnesota Institutional Review Board and by Quorum, the Institutional Review Board of our Community Partner. Informed written consent and assent was provided and collected from each participant. This paper does not include any clinical trial data. At the baseline visit, study procedures were described, questions answered and parent and child participants provided written and informed consent/assent for participation, respectively.

\section{Consent for publication}

NA

\section{Competing interests}

The authors have no known conflicts of interest as they may pertain to this study.

\section{Author details}

${ }^{1}$ Division of Epidemiology and Community Health, School of Public Health, University of Minnesota, 1300 S. 2nd St., Suite 300, Minneapolis, MN 55454, USA. ${ }^{2}$ School of Nursing, University of Minnesota, Minneapolis, MN, USA.

${ }^{3}$ Minneapolis Heart Institute Foundation, Minneapolis, MN, USA.

Received: 14 December 2020 Accepted: 15 September 2021

\section{Published online: 22 October 2021}

\section{References}

1. Hales CM, Carroll MD, Fryar CD, Ogden CL. Prevalence of Obesity Among Adults and Youth: United States, 2015-2016. NCHS data brief 2017(288):1-8.

2. Johnson JA, 3rd, Johnson AM. Urban-rural differences in childhood and adolescent obesity in the United States: a systematic review and metaanalysis. Childhood obesity (Print). 2015;11(3):233-241.

3. Davis AM, Bennett KJ, Befort C, Nollen N. Obesity and related health behaviors among urban and rural children in the United States: data from the National Health and Nutrition Examination Survey 2003-2004 and 20052006. J Pediatr Psychol. 2011;36(6):669-76. https://doi.org/10.1093/jpepsy/ jsq117.

4. United States Census Bureau. Urban and Rural. 2021; https://www.census. gov/programs-surveys/geography/guidance/geo-areas/urban-rural.html.

5. Ogden CL, Fryar CD, Hales CM, Carroll MD, Aoki Y, Freedman DS. Differences in obesity prevalence by demographics and urbanization in US children and adolescents, 2013-2016. Jama. 2018;319(23):2410-8. https://doi. org/10.1001/jama.2018.5158.

6. Strochlic $R, A u L E$, Ritchie L. Is urban-rural location associated with weight status in school children? An examination of 42 small and rural Californian counties. Rural Remote Health. 2017;17(2):3966. https://doi.org/10.22605/ $\mathrm{RRH} 3966$.

7. Ogden $\mathrm{CL}$, Carroll MD, Fakhouri TH, Hales CM, Fryar CD, Li X, et al. Prevalence of obesity among youths by household income and education level of head of household - United States 2011-2014. MMWR Morb Mortal Wkly Rep. 2018;67(6):186-9. https://doi.org/10.15585/mmwr.mm6706a3.

8. Wood SL, Demougin PR, Higgins S, Husk K, Wheeler BW, White M. Exploring the relationship between childhood obesity and proximity to the coast: a rural/urban perspective. Health \& place. 2016;40:129-36. https://doi.org/10.1 016/j.healthplace.2016.05.010.

9. Singh AS, Mulder C, Twisk JW, van Mechelen W, Chinapaw MJ. Tracking of childhood overweight into adulthood: a systematic review of the literature. Obesity reviews : an official journal of the International Association for the Study of Obesity. 2008;9(5):474-88. https://doi.org/10.1111/j.1467-789X.2008. 00475.x.

10. Bjørge T, Engeland A, Tverdal A, Smith GD. Body mass index in adolescence in relation to cause-specific mortality: a follow-up of 230,000 Norwegian adolescents. Am J Epidemiol. 2008;168(1):30-7. https://doi.org/10.1093/aje/ kwn096.

11. Robinson JC, Lim CS, Hinton E, Pintado I, Gamble A, Compretta C, et al. School-based obesity prevention programs in rural communities: a scoping review protocol. JBI Database System Rev Implement Rep. 2019;17(7):132633. https://doi.org/10.11124/JBISRIR-2017-003957.

12. Forbes CC, Yu ZM, Cui Y, DeClercq V, Grandy SA, Parker L, et al. Rural-urban disparities in Total physical activity, body composition, and related health indicators: an Atlantic PATH study. The Journal of rural health : official journal of the American Rural Health Association and the National Rural Health Care Association. 2020;36(1):111-9. https://doi.org/10.1111/jrh.12363.

13. Shah HD, Adler J, Ottoson J, Webb K, Gosliner W. Leaders' experiences in planning, implementing, and evaluating complex public health nutrition interventions. J Nutr Educ Behav. 2019;51(5):528-38. https://doi.org/10.1016/ j.jneb.2019.02.005.

14. Bolin JN, Bellamy GR, Ferdinand AO, Vuong AM, Kash BA, Schulze A, et al. Rural healthy people 2020: new decade, same challenges. The Journal of rural health : official journal of the American Rural Health Association and the National Rural Health Care Association. 2015;31(3):326-33. https://doi. org/10.1111/jrh.12116.

15. Kaczynski AT, Eberth JM, Stowe EW, Wende ME, Liese AD, McLain AC, et al. Development of a national childhood obesogenic environment index in the United States: differences by region and rurality. The international journal of behavioral nutrition and physical activity. 2020;17(1):83. https://doi.org/10.11 86/s12966-020-00984-x.

16. Foti KE, Perez CL, Knapp EA, Kharmats AY, Sharfman AS, Arteaga SS, et al. Identification of measurement needs to prevent childhood obesity in highrisk populations and environments. Am J Prev Med. 2020;59(5):746-54. https://doi.org/10.1016/j.amepre.2020.05.012.

17. Euler $R$, Jimenez EY, Sanders $S$, Kuhlemeier A, van Horn ML, Cohen $D$, et al. Rural-urban differences in baseline dietary intake and physical activity levels of adolescents. Prev Chronic Dis. 2019;16:E01. https://doi.org/10.5888/pcd1 6.180200.

18. Lynch WC, Martz J, Eldridge G, Bailey SJ, Benke C, Paul L. Childhood obesity prevention in rural settings: background, rationale, and study design of '4Health,' a parent-only intervention. BMC Public Health 2012;12:255, Childhood obesity prevention in rural settings: background, rationale, and study design of '4-Health,' a parent-only intervention, 1, DOl: https://doi. org/10.1186/1471-2458-12-255.

19. Brown CL, Halvorson EE, Cohen GM, Lazorick S, Skelton JA. Addressing childhood obesity: opportunities for prevention. Pediatr Clin N Am. 2015; 62(5):1241-61. https://doi.org/10.1016/j.pcl.2015.05.013.

20. Must A, Strauss RS. Risks and consequences of childhood and adolescent obesity. International journal of obesity and related metabolic disorders : journal of the International Association for the Study of Obesity. 1999; 23(Suppl 2):S2-11. https://doi.org/10.1038/sj.ijo.0800852.

21. Lee $A$, Cardel M, Donahoo WT. Social and environmental factors influencing obesity. In: Feingold KR, Anawalt B, Boyce a, et al., eds. Endotext. South Dartmouth (MA): MDText.com. Inc.

22. Ling J, King KM, Speck BJ, Kim S, Wu D. Preliminary assessment of a schoolbased healthy lifestyle intervention among rural elementary school children. The Journal of school health. 2014;84(4):247-55. https://doi.org/10.1111/ josh.12143.

23. Wang Y, Cai L, Wu Y, Wilson RF, Weston C, Fawole O, et al. What childhood obesity prevention programmes work? A systematic review and meta-analysis. Obesity reviews : an official journal of the International Association for the Study of Obesity. 2015;16(7):547-65. https://doi.org/10.1111/obr.12277.

24. Weihrauch-Blüher S, Kromeyer-Hauschild K, Graf C, Widhalm K, Korsten-Reck $U$, Jödicke $B$, et al. Current guidelines for obesity prevention in childhood and adolescence. Obesity facts. 2018;11(3):263-76. https://doi.org/10.1159/ 000486512.

25. Brown $T$, Moore $T H$, Hooper $L$, et al. Interventions for preventing obesity in children. The Cochrane database of systematic reviews. 2019;7(7):Cd001871.

26. Vidgen HALP, Wutzke SE, Daniels LA, Rissel CE, Innes-Hughes C, Baur LA. A description of health care system factors in the implementation of universal weight management services for children with overweight or obesity: case studies from Queensland and New South Wales. Australia Implement Sci. 2008;13(1):109. https://doi.org/10.1186/s13012-018-0801-2.

27. Croyden DL, Vidgen HA, Esdaile E, Hernandez E, Magarey A, Moores CJ, et al. A narrative account of implementation lessons learnt from the dissemination of an up-scaled state-wide child obesity management program in Australia: PEACH ${ }^{\mathrm{TM}}$ (parenting, eating and activity for child health) Queensland. BMC Public Health. 2018;18(1):347. https://doi.org/10.11 86/s12889-018-5237-8.

28. Cohen JF, Kraak VI, Choumenkovitch SF, Hyatt RR, Economos CD. The CHANGE study: a healthy-lifestyles intervention to improve rural children's diet quality. J Acad Nutr Diet. 2014;114(1):48-53. https://doi.org/10.1016/j.ja nd.2013.08.014

29. Janicke DM, Lim CS, Perri MG, Mathews AE, Bobroff LB, Gurka MJ, et al. Featured article: behavior interventions addressing obesity in rural settings: 
the E-FLIP for kids trial. J Pediatr Psychol. 2019;44(8):889-901. https://doi. org/10.1093/jpepsy/jsz029.

30. Ward M, Schulz AJ, Israel BA, Rice K, Martenies SE, Markarian E. A conceptual framework for evaluating health equity promotion within community-based participatory research partnerships. Evaluation and program planning. 2018; 70:25-34. https://doi.org/10.1016/j.evalprogplan.2018.04.014

31. Birch LL, Davison KK. Family environmental factors influencing the developing behavioral controls of food intake and childhood overweight. Pediatr Clin N Am. 2001;48(4):893-907. https://doi.org/10.1016/50031-3 955(05)70347-3.

32. Tong A, Sainsbury P, Craig J. Consolidated criteria for reporting qualitative research (COREQ): a 32-item checklist for interviews and focus groups. Int $J$ Qual Health Care. 2007;19(6):349-57. https://doi.org/10.1093/intahc/ mzm042.

33. Chambers DA, Norton WE. The Adaptome: advancing the science of intervention adaptation. Am J Prev Med. 2016;51(4 Suppl 2):S124-31. https://doi.org/10.1016/j.amepre.2016.05.011.

34. Fulkerson JA, Neumark-Sztainer D, Story M, Gurvich O, Kubik MY, Garwick A, et al. The healthy home offerings via the mealtime environment (HOME) plus study: design and methods. Contemporary clinical trials. 2014;38(1):5968. https://doi.org/10.1016/j.cct.2014.01.006.

35. Fulkerson JA, Rydell S, Kubik MY, et al. Healthy Home Offerings via the Mealtime Environment (HOME): feasibility, acceptability, and outcomes of a pilot study. Obesity (Silver Spring, Md). 2010;18 Suppl 1(Suppl 1):S69-74.

36. Flattum C, Draxten M, Horning M, Fulkerson JA, Neumark-Sztainer D, Garwick A, et al. HOME plus: program design and implementation of a family-focused, community-based intervention to promote the frequency and healthfulness of family meals, reduce children's sedentary behavior, and prevent obesity. The international journal of behavioral nutrition and physical activity. 2015;12(1):53. https://doi.org/10.1186/s12966-015-0211-7.

37. Fulkerson JA, Friend $\mathrm{S}$, Flattum $\mathrm{C}$, Horning M, Draxten M, Neumark-Sztainer $D$, et al. Promoting healthful family meals to prevent obesity: HOME plus, a randomized controlled trial. The international journal of behavioral nutrition and physical activity. 2015;12(1):154. https://doi.org/10.1186/s12966-015-032 0-3.

38. Fulkerson JA, Friend S, Horning M, Flattum C, Draxten M, Neumark-Sztainer D, et al. Family home food environment and nutrition-related parent and child personal and behavioral outcomes of the healthy home offerings via the mealtime environment (HOME) plus program: a randomized controlled trial. Acad Nutr Diet. 2018;1 18(2):240-51. https://doi.org/10.1016/j.jand.2017.04.006.

39. Sidebottom AC, Sillah A, Miedema MD, Vock DM, Pereira R, Benson G, et al. Changes in cardiovascular risk factors after 5 years of implementation of a population-based program to reduce cardiovascular disease: the heart of New Ulm project. Am Heart J. 2016;175:66-76. https://doi.org/10.1016/j.ahj.2016.02.006.

40. VanWormer JJ, Johnson PJ, Pereira RF, et al. The heart of New Ulm project: using community-based cardiometabolic risk factor screenings in a rural population health improvement initiative. Population health management. 2012;15(3):135-43. https://doi.org/10.1089/pop.2011.0027.

41. Boucher JL, Pereira RF, Graham KJ, Pettingill RR, Toscano JV, Henry TD. The heart of New Ulm: a vision for the future. J Cardiovasc Transl Res. 2008; 1(4): 310-6. https://doi.org/10.1007/s12265-008-9077-2.

42. Fulkerson JA, Horning ML, Barr-Anderson DJ, Linde JA, Sidebottom AC, Lindberg $R$, et al. Universal childhood obesity prevention in a rural community: study design, methods and baseline participant characteristics of the NU-HOME randomized controlled trial. Contemporary clinical trials. 2021;100:106160. https://doi.org/10.1016/j.cct.2020.106160.

43. Cassidy O, Sbrocco T, Vannucci A, Nelson B, Jackson-Bowen D, Heimdal J, et al. Adapting interpersonal psychotherapy for the prevention of excessive weight gain in rural African American girls. J Pediatr Psychol. 2013;38(9): 965-77. https://doi.org/10.1093/jpepsy/jst029.

44. Connell CL, Thomson JL, Huye HF, Landry AS, Crook LB, Yadrick K. Mississippi communities for healthy living: implementing a nutrition intervention effectiveness study in a rural health disparate region. Contemporary clinical trials. 2015;42:196-203. https://doi.org/10.1016/j.cct.2 015.04.003.

45. Shea CM, Young TL, Powell BJ, Rohweder C, Enga ZK, Scott JE, et al. Researcher readiness for participating in community-engaged dissemination and implementation research: a conceptual framework of core competencies. Transl Behav Med. 2017;7(3):393-404. https://doi.org/10.1007/ s13142-017-0486-0.
46. Skinner JS, Williams NA, Richmond A, Brown J, Strelnick AH, Calhoun K, et al. Community experiences and perceptions of clinical and translational research and researchers. Progress in community health partnerships: research, education, and action. 2018;12(3):263-71. https://doi.org/10.1353/ cpr.2018.0050.

47. Vaughn LM, Jacquez F, Zhen-Duan J. Perspectives of community coresearchers about group dynamics and equitable partnership within a community-academic research team. Health education \& behavior : the official publication of the Society for Public Health Education. 2018;45(5): 682-9. https://doi.org/10.1177/1090198118769374.

48. Mensah GA, Cooper RS, Siega-Riz AM, Cooper LA, Smith JD, Brown CH, et al. Reducing cardiovascular disparities through community-engaged implementation research: a National Heart, Lung, and Blood Institute workshop report. Circ Res. 2018;122(2):213-30. https://doi.org/10.1161/ CIRCRESAHA. 117.312243

49. Israel BA, Coombe CM, Cheezum RR, Schulz AJ, McGranaghan RJ, Lichtenstein $\mathrm{R}$, et al. Community-based participatory research: a capacitybuilding approach for policy advocacy aimed at eliminating health disparities. Am J Public Health. 2010;100(11):2094-102. https://doi.org/10.21 05/A.PH.2009.170506.

50. Coombe CM, Schulz AJ, Guluma L, Allen AJ III, Gray C, Brakefield-Caldwell W, et al. Enhancing capacity of community-academic partnerships to achieve health equity: results from the CBPR partnership academy. Health Promot Pract. 2020;21(4):552-63. https://doi.org/10.1177/1524839918818830.

51. Ells LJ, Rees K, Brown T, Mead E, al-Khudairy L, Azevedo L, et al. Interventions for treating children and adolescents with overweight and obesity: an overview of Cochrane reviews. Int J Obes. 2018;42(11):1823-33. https://doi.org/10.1038/s41366-018-0230-y.

52. Martin CL, Kramer-Kostecka EN, Linde JA, Friend S, Zuroski VR, Fulkerson JA. Leveraging interdisciplinary teams to develop and implement secure websites for behavioral research: applied tutorial. J Med Internet Res. 2020; 22(9):e19217. https://doi.org/10.2196/19217.

53. White AA, Colby SE, Franzen-Castle L, Kattelmann KK, Olfert MD, Gould TA, et al. The iCook 4-H study: an intervention and dissemination test of a youth/adult out-of-school program. J Nutr Educ Behav. 2019;51(3s):S2-s20. https://doi.org/10.1016/j.jneb.2018.11.012.

54. Hawley SR, Beckman H, Bishop T. Development of an obesity prevention and management program for children and adolescents in a rural setting. J Community Health Nurs. 2006;23(2):69-80. https://doi.org/10.1207/s1532 7655jchn2302_1.

55. Health Resources and Services Administration (HRSA) of the U.S. Department of Health and Human Services (HHS). Rural Health Information Hub, 2002-2020. . 2020; https://www.ruralhealthinfo.org/topics/socialdeterminants-of-health\#rural-difference, 2020

56. Smith JD, Berkel C, Rudo-Stern J, Montaño Z, St. George SM, Prado G, et al. The family check-up 4 health (FCU4Health): applying implementation science frameworks to the process of adapting an evidence-based parenting program for prevention of pediatric obesity and excess weight gain in primary care. Front Public Health. 2018;6:293. https://doi.org/10.33 89/fpubh.2018.00293.

57. Bergling E, Farewell C, Puma J. Development of a dissemination and implementation framework for an early childhood obesity prevention program. J Nutr Educ Behav. 2020;52(12):1160-5. https://doi.org/10.1016/j. jneb.2020.08.012.

58. Pérez-Escamilla R, Vilar-Compte $M$, Rhodes $E$, et al. Implementation of childhood obesity prevention and control policies in the United States and Latin America: Lessons for cross-border research and practice. Obesity reviews : an official journal of the International Association for the Study of Obesity. 2021:e13247.

\section{Publisher's Note}

Springer Nature remains neutral with regard to jurisdictional claims in published maps and institutional affiliations. 